\title{
Rosuvastatin as a potential preventive drug for the development of hepatocellular carcinoma associated with non-alcoholic fatty liver disease in mice
}

\author{
KEISUKE YOKOHAMA ${ }^{1}$, SHINYA FUKUNISHI ${ }^{1}$, MASAAKI II ${ }^{2}$, KEN NAKAMURA ${ }^{1}$, HIDEKO OHAMA ${ }^{1}$, \\ YUSUKE TSUCHIMOTO $^{1}$, AKIRA ASAI ${ }^{1}$, YASUHIRO TSUDA ${ }^{1}$ and KAZUHIDE HIGUCHI ${ }^{1}$ \\ ${ }^{1}$ Second Department of Internal Medicine, and ${ }^{2}$ Department of Pharmacology, \\ Osaka Medical College, Takatsuki, Osaka 569-8686, Japan
}

Received May 9, 2016; Accepted September 12, 2016

DOI: 10.3892/ijmm.2016.2766

\begin{abstract}
Hepatocellular carcinoma (HCC) represents approximately $85 \%$ of all primary liver cancer cases. Non-alcoholic fatty liver disease (NAFLD) is one of the risk factors for HCC. NAFLD occurs in patients with components of metabolic syndrome, such as type 2 diabetes mellitus, obesity, hypertension and hyperlipidemia. Therefore, hyperlipidemia also represents a patient population at risk for HCC that can readily be identified. Rosuvastatin, a 3-hydroxy-3-methyl-glutaryl coenzyme A (HMG-CoA) reductase inhibitor, has exhibited a more potent affinity for the active site of HMG-CoA reductase than other statins. In addition, the hepatic uptake of rosuvastatin in rats has been found to be more selective and efficient than that with other drugs. Furthermore, the cytoprotective effects of rosuvastatin against ischemic injury have been clearly reported.
\end{abstract}

Correspondence to: Dr Shinya Fukunishi, Second Department of Internal Medicine, Osaka Medical College 2-7 Daigaku-machi, Takatsuki, Osaka 569-8686, Japan

E-mail: in2104@osaka-med.ac.jp

Abbreviations: HCC, hepatocellular carcinoma; NAFLD, nonalcoholic fatty liver disease; HMG-CoA, 3-hydroxy-3-methylglutaryl coenzyme A; TNF, tumor necrosis factor; IL, interleukin; TGF, transforming growth factor; VEGFR, vascular endothelial growth factor receptor; EGFR, epidermal growth factor receptor; PDGF, platelet-derived growth factor; NASH, non-alcoholic steatohepatitis; Ros, Rosuvastatin; HFD, high-fat-diet; AST, spartate aminotransferase; ALT, alanine aminotransferase; FFA, free fatty acid; TG, triglyceride; T-CHO, total cholesterol; TG, tissue triglyceride; H\&E hematoxylin and eosin; RT-qPCR, reverse transcription-quantitative PCR; IFN, interferon; SREBP-1c, sterol regulatory element binding protein-1c; AMPK, AMP-activated protein kinase; FAS, fatty acid synthase; SCD-1, stearoyl-CoA desaturase-1; PPAR, peroxisome proliferator-activated receptor; HSCs, hepatic stellate cells; TIMP, tissue inhibitors of matrix metalloproteinases; MMP, matrix metalloproteinases; Gclc, GCL catalytic subunit; GST, glutathione S-transferase; EPA, eicosapentaenoic acid

Key words: hepatocellular carcinoma, non-alcoholic fatty liver disease, rosuvastatin, mice, non-alcoholic steatohepatitis
Thus, in this study, we aimed to determine the role of rosuvastatin as a preventive drug in HCC associated with NAFLD. STAM mice, which developed HCC from NAFLD by being fed a high-fat diet (HFD), were divided into a group in which a HFD was given to the mice for 15 weeks $(n=8)$ and another in which a HFD supplemented with $0.00125 \%$ rosuvastatin was given to the mice for 15 weeks $(n=8)$. Rosuvastatin inhibited the development of hepatic tumors in the mice with NAFLD induced by a specific diet both macroscopically and histologically. Rosuvastatin significantly decreased the expression levels of pro-inflammatry cytokines, such as tumor necrosis factor (TNF)- $\alpha$, interleukin (IL)- 6 and transforming growth factor (TGF)- $\beta 1$. Tumor aggressiveness is mediated by angiogenic factors. Therefore, we examined the hepatic mRNA expression of vascular endothelial growth factor receptor (VEGFR), epidermal growth factor receptor (EGFR) and platelet-derived growth factor (PDGF). The hepatic expression of these factors significantly decreased in the rousvastin-fed mice. Our results thus suggest rosuvastatin that prevents carcinogenesis and improves the hepatic background. Our data suggest that rosuvastatin has potential for use as a preventive drug for the development of HCC associated with NAFLD in mice.

\section{Introduction}

Primary liver cancer is fifth most common type of cancer and has the third highest rates of mortality worldwide. Hepatocellular carcinoma (HCC) represents approximately $85 \%$ of all primary liver cancer cases. Although the incidence of some types of cancer is decreasing, the incidence of HCC is increasing worldwide $(1,2)$. A number of risk factors have been identified to increase the risk of HCC. Non-alcoholic fatty liver disease (NAFLD) is one of these risk factors. The histological changes occurring in NAFLD range over a wide spectrum, extending from simple steatosis to non-alcoholic steatohepatitis (NASH), liver cirrhosis and liver failure, and sometimes even HCC (3). NAFLD occurs in patients with components of metabolic syndrome, such as type 2 diabetes mellitus (T2DM), obesity, hypertension and hyperlipidemia (3). Therefore, hyperlipidemia also represents a patient population at risk for HCC that can readily be identified. 
Statins are effective drug for patients with hyperlipidemia. The drugs inhibit 3-hydroxy-3-methyl-glutaryl coenzyme A (HMG-CoA) reductase, a key enzyme that catalyzes the rate-limiting step within the cholesterol biosynthetic pathway. Previous studies have shown that statins can exert effects separate their lipid-lowering properties. These non-lipid effects include antioxidant effects (4), anti-inflammatory effects (5) and the upregulation of endothelial nitric oxide synthase (6). Rosuvastatin (Ros), a HMG-CoA reductase inhibitor, has exhibited a more potent affinity for the active site of HMG-CoA reductase than other statins. In addition, the hepatic uptake of Ros in rats has been found to be more selective and efficient than that with other drugs (7). Furthermore, the cytoprotective effects of Ros against ischemic injury have been clearly reported (8-11). Thus, in this study, we aimed to determine the role of Ros as a preventive drug in HCC associated with NAFLD.

\section{Materials and methods}

Chemicals and diets. Rosuvastatin Calcium was purchased from AvaChem Scientific (San Antonio, TX, USA). The experimental diets [high-fat-diet (HFD)] were purchased from Oriental Yeast Co., Ltd. (Tokyo, Japan). The energy content of the diet was $5.1 \mathrm{kcal} / \mathrm{g}$, with $56.7 \%$ of calories from fat, $20.1 \%$ of calories from protein, and $23.2 \%$ of calories from carbohydrate, plus vitamins and minerals as recommended. The diets were freshly prepared each day. Ros was administered orally by premixing with the HFD to a concentration of $0.00125 \%$ to a concentration of $0.00125 \%$, as these concentrations of the drug have been administered to patients with hyperlipidemia.

Animals. STAM mice, a NASH-cirrhosis-hepatocarcinogenic model, were purchased from STELIC Co., Ltd. (Tokyo, Japan). The mouse model was established according to a previously described protocol (12). Briefly, pregnant C57BL/6 mice were purchased from CLEA-Japan (Tokyo, Japan) and 2-day-old male pups were injected with streptozotocin (200 $\mu \mathrm{g} / \mathrm{mouse})$ and fed a HFD (HFD-32; CLEA-Japan) from the age of 4 weeks. This mouse model progresses from NAFLD to $\mathrm{NASH}$ at 8 weeks of age and develops HCC at 16 weeks of age (12).

Experimental design. After weaning, 16 mice were divided into 2 experimental groups. The experimental designs were as follows: 5-week-old male STAM mice, which developed T2DM and NAFLD by being fed a HFD, were divided into a group in which a HFD was given to the mice for 15 weeks $(n=8)$ as controls (HFD group); in the other group, the mice were fed a HFD supplemented with $0.00125 \%$ Ros for 15 weeks $(n=8)$ (Ros group). The mice were allowed free access to food, with a 12-h light/12-h dark cycle under conditions of controlled temperature $\left(22 \pm 1^{\circ} \mathrm{C}\right)$ and humidity $(50 \pm 10 \%)$. Food intake was measured daily, while individual body weight was recorded once a week. The 8 mice from each group were fasted overnight prior to euthanasia (by cervical dislocation). All mice were sacrificed after completing their respective dietary regimens, and the livers of the individual animals were weighed. The livers were removed, the samples were placed in formalin and the remainder were snap-frozen and stored at $-80^{\circ} \mathrm{C}$. All surgical and experimental procedures were performed according to the guidelines for the care and use of animals and approved by the Osaka Medical College Ethics Committee.

Assay for plasma hepatic and metabolic parameters. Blood samples were obtained by cardiac puncture and separated by centrifugation $(12,000 \mathrm{rpm}, 15 \mathrm{~min})$ as plasma. The levels of blood biochemical parameters, including aspartate aminotransferase (AST), alanine aminotransferase (ALT), free fatty acid (FFA), triglyceride (TG) and total cholesterol (T-CHO) were measured by a local laboratory specified in clinical analyses (Oriental Yeast Co., Ltd.).

Assay for hepatic lipid content. The hepatic tissues were homogenized using a Janke \& Kunkel Polytron homogenizer (Ultra-Turrax TP18/1051; IKA Labortechnik, Staufen, Germany) in buffer ( $\mathrm{pH}$ 7.4) containing $20 \mathrm{mM}$ Tris-HCl, $1 \mathrm{mM}$ EGTA, $2 \mathrm{mM}$ EDTA, and treated with protease inhibitor $(2 \mu \mathrm{g} / \mathrm{ml}$, leupeptin cocktail). Hepatic TG levels were measured by a local laboratory that specifies in clinical analyses (SRL Co. Ltd., Tokyo, Japan).

Histological analysis of hepatic tissue. The liver sections were examined blindly from different lobes of each mouse. Liver tissues were fixed in $10 \%$ buffered formaldehyde, and then embedded in paraffin. A 4-mm-thick section cut from a paraffin-embedded block was stained with hematoxylin and eosin (H\&E; Applied Medical Research, Osaka, Japan).

Reverse transcription-quantitative PCR (RT-qPCR). Tissue specimens were preserved in RNAlater reagent (Qiagen, Valencia, CA, USA) until the isolation of the total RNA. Total RNA was isolated from the liver tissues using a QIAshredder and an RNeasy kit (Qiagen). cDNA was prepared using the TaqMan reverse transcriptase kitQiagen (Qiagen). Quantitative (real-time) PCR (qPCR) was performed using the StrataScript First Strand cDNA synthesis kit and FullVelocity SYBR-Green qPCR Master Mix (Stratagene, La Jolla, CA, USA) according to the manufacturer's instructions. The primers used for qPCR were designed using Beagon Designer software version 2.12 , according to the parameters outlined in the Bio-Rad iCycler Manual, using reference mRNA sequences accessed through GenBank and as shown in Table I. All probes used in the TaqMan Gene Expression assays were purchased from Applied Biosystems (Foster City, CA, USA). PCR reactions were carried out in the iCycler Thermal Cycler (Bio-Rad Laboratories, Hercules, CA, USA). PCR products were detected using the iCycler IQ Real-Time PCR detection system (Bio-Rad). The relative amount of mRNA was calculated by comparative cycle time determination with the ribosomal protein, RPL32 as the invariant control. Gene expression values were calculated based on the $\Delta \Delta \mathrm{Ct}$ method. The results were expressed as a fold increase in expression relative to the control group.

Statistical analysis. Data are presented as the means \pm standard error of the mean. Statistical analyses were performed using Student's t-test. Values of $\mathrm{p}<0.05$ were considered to indicate statistically significant differences. 
Table I. Sequences of primers used for the RT-qPCR.

\begin{tabular}{|c|c|c|}
\hline Gene & Primer sequences (sense) & Primer sequences (antisense) \\
\hline $\mathrm{TNF}-\alpha$ & 5'-ACCTTGTTGCCTCCTCTT-3' & 5'-GTTCAGTGATGTAGCGACAG-3' \\
\hline IL-1 $\beta$ & 5'-TCCAGGATGAGGACATGAGCAC-3' & 5'-GAACGTCACACACCAGCAGGTTA-3' \\
\hline IL-6 & 5'-TTCCTCACTGTGGTCAGA-3' & 5'-CATTCATATTGTCAGTTCTTCGTA-3' \\
\hline IFN- $\gamma$ & 5'-CGGCACAGTCATTGAAAGCCTA-3' & 5'-GTTGCTGATGGCCTGATTGTC-3' \\
\hline SREBP-1c & 5'-GGTACCTGCGGGACAGCTTA-3' & 5'-CCGTGAGCTACCTGGACTGAA-3' \\
\hline FAS & 5'-TACAGATGGCAGCAAGGA-3' & 5'-TGATACAGAGAGCAGATGAGT-3' \\
\hline PPAR- $\alpha$ & 5'-ATGGCAGCAATATCAGAG-3' & 5'-AGCAGTAAAGTATCATATCAAAG-3' \\
\hline PPAR $-\gamma$ & 5'-GAAGACAGAGACAGACAT-3' & 5'-GCAATCAATAGAAGGAACA-3' \\
\hline SCD-1 & 5'-CTGGCTGGAGAGTCATCA-3' & 5'-TAACGAGGACGACAATACAATC-3' \\
\hline AMPK $\alpha 1$ & 5'-CCTTCGGGAAAGTGAAGGT-3' & 5'-GAATCTTCTGCCGGTTGAGT-3' \\
\hline Gclc & 5'-ATGATAGAACACGGGAGGAGAG-3' & 5'-TGATCCTAAAGCGATTGTTCTTC-3' \\
\hline GST & 5'-CСТCCCCACAGTGAAGAAGT-3' & 5'-CAACACATTTTGCGTCATCA-3' \\
\hline TGF- $\beta 1$ & 5'-GCGATACCTCAGCAACCG-3' & 5'-CTAAGGCGAAAGCCCTCAAT-3' \\
\hline TIMP1 & 5'-GCATCTCTGGCATCTGGCATC-3' & 5'-GCGGTTCTGGGACTTGTGGGC-3' \\
\hline MMP-13 & 5'-CCTTCTGGTCTTCTGGCACAC-3' & 5'-GGCTGGGTCACACTTCTCTGG-3' \\
\hline Type 1 collagen $\alpha 1$ & 5'-ACCTGTGTGTTCCCTACTCA-3' & 5'-GACTGTTGCCTTCGCCTCTG-3' \\
\hline EGFR & 5'-TCTTCAAGGATGTGAAGTGTG-3' & 5'-TGTACGCTTTCGAACAATGT-3' \\
\hline VEGFR & 5'-TACTGCTGTACCTCCACCTCCACCATG-3' & 5'-TCACTTCATGGGACTTCTGCTCT-3' \\
\hline PDGFR & 5'-TCСТTCTACCACCTCAGCGAG-3' & 5'-CCGGATGGTCACTCTTTAGGAAG-3' \\
\hline
\end{tabular}

TNF- $\alpha$, tumor necrosis factor- $\alpha$; IL, interleukin; IFN- $\gamma$, interferon- $\alpha$; SREBP-1c, sterol regulatory element binding protein- $1 \mathrm{c}$; FAS, fatty acid synthase; PPAR, peroxisome proliferator-activated receptor; SCD-1, stearoyl-CoA desaturase-1; AMPK, AMP-activated protein kinase; Gclc, GCL catalytic subunit; GST, glutathione S-transferase; TGF- $\beta 1$, transforming growth factor- $\beta 1$; TIMP1, tissue inhibitors of matrix metalloproteinase 1; MMP-13, matrix metalloproteinase 13; EGFR, epidermal growth factor receptor; VEGFR, vascular endothelial growth factor receptor; PDGFR, platelet-derived growth factor receptor.

Table II. Plasma and hepatic biochemical parameters and liver weight/body weight ratios of the HFD group and Ros group.

\begin{tabular}{lcc}
\hline Parameter & $\begin{array}{c}\text { HFD group } \\
(\mathrm{n}=8)\end{array}$ & $\begin{array}{c}\text { Ros group } \\
(\mathrm{n}=8)\end{array}$ \\
\hline Liver/body weight & $7.2 \pm 0.56$ & $7.5 \pm 0.04$ \\
ratio $(\%)$ & & \\
AST (IU/l) & $189.75 \pm 441.19$ & $138.13 \pm 42.84^{\mathrm{a}}$ \\
ALT $(\mathrm{IU} / \mathrm{l})$ & $59.88 \pm 20.32$ & $39.75 \pm 6.92^{\mathrm{a}}$ \\
T-CHO (mg/dl) & $161.88 \pm 52.14$ & $109.13 \pm 14.64^{\mathrm{a}}$ \\
FFA $(\mu \mathrm{Eq} / \mathrm{l})$ & $514.5 \pm 144.15$ & $350.75 \pm 119.7^{\mathrm{a}}$ \\
TG $(\mathrm{mg} / \mathrm{dl})$ & $150.13 \pm 68.16$ & $39.0 \pm 8.29^{\mathrm{a}}$ \\
Hepatic TG $(\mathrm{mg} / \mathrm{dl})$ & $38.11 \pm 2.44$ & $29.99 \pm 1.22^{\mathrm{a}}$ \\
\hline
\end{tabular}

${ }^{\mathrm{a}} \mathrm{p}<0.05$ vs. HFD group. HFD, high-fat-diet; AST, aspartate aminotransferase; ALT, alanine aminotransferase; T-CHO, total cholesterol; FFA, free fatty acid; TG, triglyceride.

\section{Results}

Effect of diets on the liver/body weight ratio of the mice in each experimental group. As shown Table II, the ratio of liver weight to body weight did not differ significantly between the 2 groups of mice $(7.2 \pm 0.56$ and $7.5 \pm 0.04 \%$ in the HFD and Ros group, respectively; $\mathrm{p}<0.05)$.
Plasma and hepatic biochemical parameters. To examine whether Ros, as a preventive drug for the development of HCC associated with NAFLD, affected liver damage and steatosis in our mouse experimental groups, we quantified the plasma levels of AST, ALT, T-CHO, TG and FFA. The plasma AST (HFD, $189.75 \pm 441.19$ vs. Ros, $138.13 \pm 42.84$ IU/1; $\mathrm{p}<0.05$ ) and ALT levels (HFD, 59.88 \pm 20.32 vs. Ros, 39.75 \pm 6.92 IU/1; $\mathrm{p}<0.05)$ differed significantly between the Ros group and HFD group (Table II). Mice fed the diet containing Ros had lower plasma levels of T-CHO (HFD, 161.88 \pm 52.14 vs. Ros, $109.13 \pm 14.64 \mathrm{mg} / \mathrm{dl} ; \mathrm{p}<0.05)$, FFA (HFD, 514.5 \pm 144.15 vs. Ros, $350.75 \pm 119.7 \mu \mathrm{Eq} / \mathrm{l} ; \mathrm{p}<0.05)$ and TG (HFD, $150.13 \pm 68.16$ vs. Ros, $39.0 \pm 8.29 \mathrm{mg} / \mathrm{dl}$; $\mathrm{p}<0.05$ ) (Table II). Mice fed the diet containing Ros also had a lower hepatic TG content (HFD, $38.11 \pm 2.44$ vs. Ros, 29.99 $\pm 1.22 \mathrm{mg} / \mathrm{dl}$; p<0.05) (Table II).

Macroscopic examinations. Numerous tumors on the liver surface were observed in 4 out of 5 mice in the HFD group (Fig. 1). On the other hand, no tumors on the liver surface were observed in the mice in the Ros group (Fig. 1).

Histological analysis. Mild hepatic steatosis was observed in the 2 groups (Fig. 2). Hepatic steatosis was however, decreased in the Ros group compared to the HFD group. Although large fatty droplets were observed in the HFD group, no large fatty droplets were observed in the Ros group. Histological findings in the liver of two groups did not show clear inflammatory 


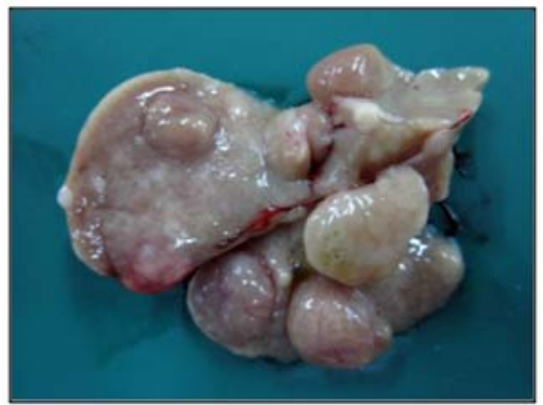

HFD group

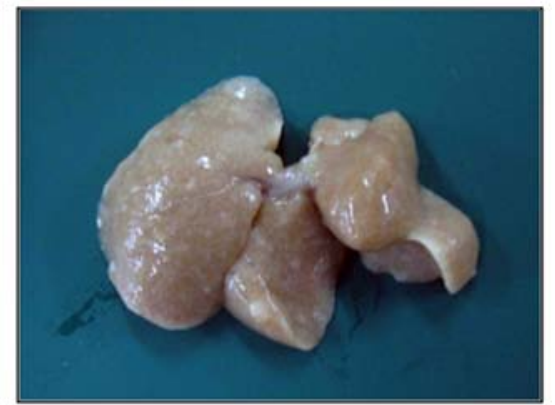

Ros group

\begin{tabular}{lcc}
\hline & HFD group $(\mathrm{n}=8)$ & Ros group $(\mathrm{n}=8)$ \\
\hline Tumor on liver surface $(\%)$ & $7 / 8(87.5)$ & $0 / 8(0)$ \\
\hline
\end{tabular}

Figure 1. Macroscopic views of a liver of a 20-week-old STAM mouse fed a high-fat-diet (HFD) or that of a mouse fed a HFD supplemented with $0.00125 \%$ rosuvastatin (Ros) (upper panel). The HFD group mouse exhibited numerous tumors on the liver surface. On the other hand, the Ros group mouse exhibited no liver tumors. The HFD group mice exhibited a significantly higher incidence (7/8: 87.5\%) of HCC than the Ros group (0/8: 0\%) at 20 weeks (lower panel). The numbers are expressed as the numbers of mice and percentage.

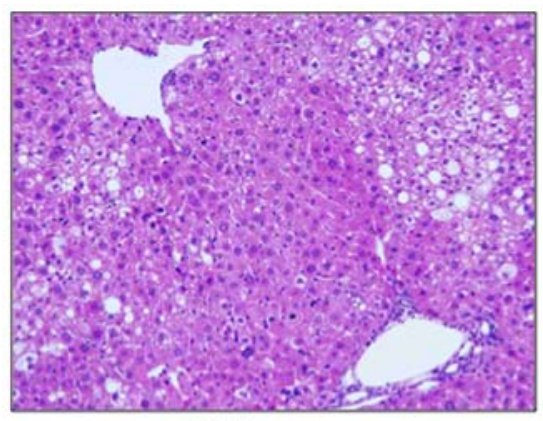

HFD group

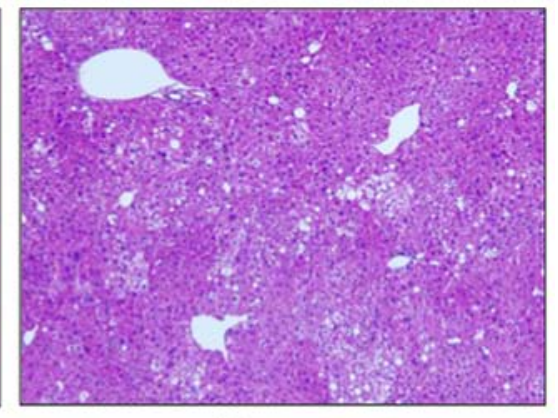

Ros group

Figure 2. Microscopic views of the liver of a 20-week-old STAM mouse fed a high-fat-diet (HFD) diet or that of a mouse fed a HFD supplemented with 0.00125\% rosuvastatin (Ros). Hepatic steatosis was decreased in the Ros group compared to the HFD group. Although large fatty droplets were observed in the HFD group, no large fatty droplets were observed in the Ros group. Histological findings in the liver of two groups did not show clear inflammatory cell infiltration.

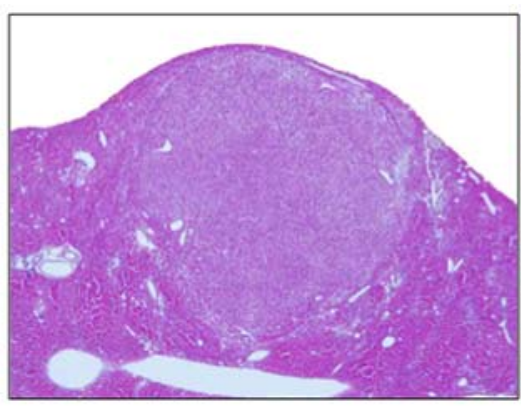

HFD group

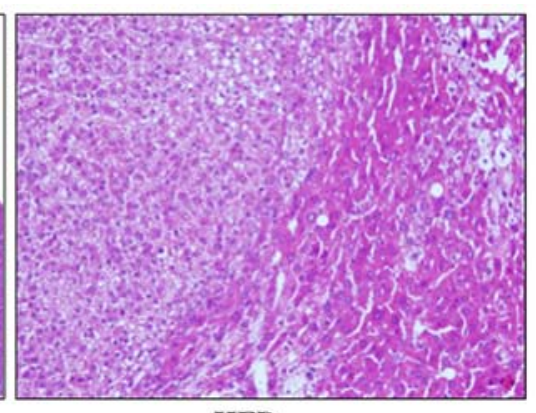

HFD group

Figure 3. Microscopic views of the liver of a 20-week-old STAM mouse fed a high-fat-diet (HFD) diet or of a mouse fed a HFD supplemented with $0.00125 \%$ rosuvastatin (Ros). Liver tumors observed in the HFD group ( $\mathrm{n}=8$ mice/group) and the rosuvastatin (Ros) group ( $\mathrm{n}=8$ mice/group). Histological finding of the livers from HFD group showing hepatocellular carcinomas (HCCs) (original magnification, $\mathrm{x} 40$ and $\mathrm{x} 100$ ).

cell infiltration. The cells in the tumor in HFD group had high nuclear/cytoplasmic ratio, and this finding did not contradict the findings of HCC. These cells were well differentiated. Therefore, histological findings revealed that the tumors were HCC (Fig. 3). On the other hand, histological examinations did not reveal any HCCs in the Ros group (Fig. 2).

Hepatic pro-inflammatory mRNA expression. A previous study demonstrated that several pro-inflammatory cytokines are associated with the development of NASH (13). Therefore, we examined the expression levels of hepatic pro-inflammatory cytokines. The relative hepatic mRNA expression levels of tumor necrosis factor (TNF)- $\alpha$, interleukin (IL)-1 $\beta$, IL-6 and interferon (IFN)- $\gamma$ were significantly decreased in the Ros group compared with the HFD group (all p<0.05) (Fig. 4).

Hepatic lipogenic-related $m R N A$ expression. The differences in the levels of plasma TG and FFA between the 2 groups and 

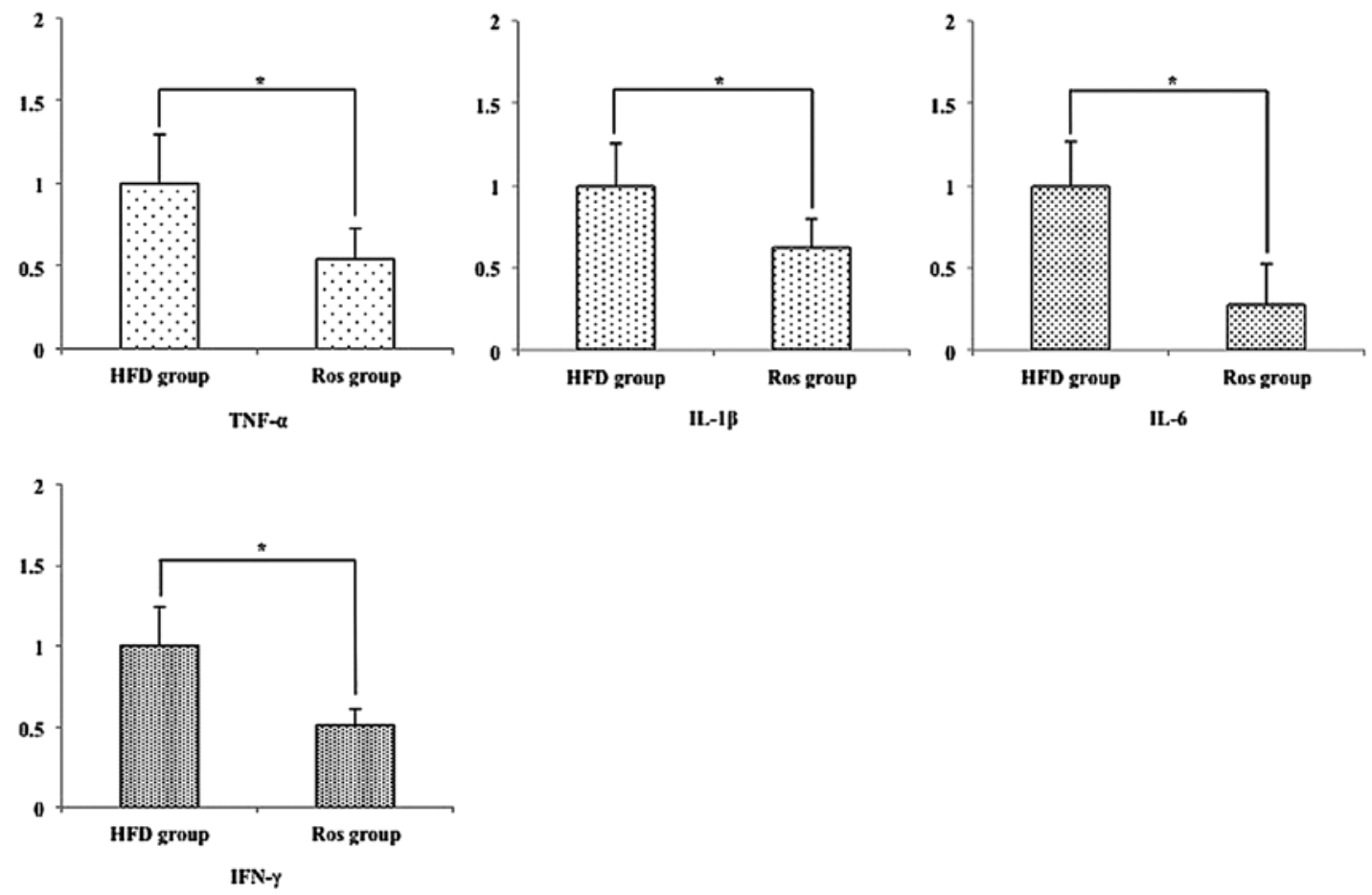

Figure 4. Relative hepatic tumor necrosis factor (TNF)- $\alpha$, interleukin (IL)-1 $\beta$, IL-6 and interferon (IFN)- $\gamma$ levels in the mice in the high-fat diet (HFD) group and in the mice in the rosuvastatin (Ros; fed the HFD supplemented with $0.00125 \%$ Ros) group were measured by RT-qPCR. The levels of TNF- $\alpha$, IL-1 $\beta$, IL-6 and IFN $-\gamma$ were significantly decreased in the rosuvastatin (Ros) group compared with the HFD group ( $\mathrm{p}<0.05)$.

the effect of Ros on the development of NAFLD suggest the expression of cytokines involved in the development of NAFLD. Sterol regulatory element binding protein-1c (SREBP-1c) is well known to be involved in these states (14). The relative hepatic mRNA expression levels of SREBP-1c were significantly decreased in the Ros group compared with the HFD group ( $p<0.05$; Fig. 5). Furthermore, it has been reported that SREBP-1 is regulated by a pathway of AMP-activated protein kinase (AMPK) (15). Since fatty acid synthase (FAS) and stearoyl-CoA desaturase-1 (SCD-1) may be critical to the role of triglyceride accumulation in hepatocytes (16), we examined the expression levels of these 3 genes. The relative hepatic mRNA expression levels of FAS were significantly decreased in the Ros group compared with the HFD group. However, there was no significant difference in the hepatic mRNA expression levels of SCD-1 between the 2 groups (Fig. 5). Peroxisome proliferatoractivated receptors (PPARs) are nuclear transcription factors that include 3 subtypes: $\alpha, \beta$ and $\gamma$. PPAR- $\alpha$ is a member of the PPAR subfamily of nuclear receptors that transcriptionally promotes peroxisomal, microsomal and mitochondrial oxidation (17). PPAR- $\gamma$, another member of the PPAR subfamily of nuclear receptors, transcriptionally activates adipocyte differentiation (18). Thus, we examined the hepatic mRNA expression levels of these genes. The relative hepatic mRNA expression levels of PPAR- $\alpha$ were significantly increased in the Ros group compared with the HFD group (Fig. 5). On the other hand, there was no significant difference in the relative hepatic mRNA expression levels of PPAR- $\gamma$ between the 2 groups (Fig. 5).

Hepatic pro-fibrogenic $m R N A$ expression. Transforming growth factor (TGF)- $\beta 1$ is produced by Kupffer cells and activates hepatic stellate cells (HSCs) that play a role in fibro- genesis in the liver (19). Thus, we examined the expression levels of TGF- $\beta 1$ and fibrogenesis-related genes produced from HSCs, such tissue inhibitors of matrix metalloproteinase (TIMP)-1, matrix metalloproteinase (MMP)-13 and type 1 collagen $\alpha 1$ (19). The relative hepatic mRNA expression levels of TGF- $\beta 1$ were significantly decreased in the Ros group compared with the HFD group (Fig. 6). However, there was no significant difference in the relative mRNA expression levels of the other genes between the 2 groups (Fig. 6).

Hepatic antioxidant-related mRNA expression. To determine whether Ros detoxifies reactive oxygen species in the Ros group, we then performed RT-qPCR to quantify the expression levels of antoxidant genes, such as GCL catalytic subunit (Gclc) and glutathione S-transferase (GST) (20). The relative hepatic expression of these genes did not differ significantly between the 2 groups (Fig. 7).

Hepatic developing HCC-related mRNA expression. Sorafenib is a useful drug for the treatment of $\mathrm{HCC}$ as it inhibits the epidermal growth factor (EGF), vascular endothelial growth factor (VEGF) and platelet-derived growth factor (PDGF) $(21,22)$. Thus, we examined whether Ros inhibits the development of these genes. The relative hepatic mRNA expression levels of EGFR, VEGER and PDGFR were significantly decreased in the Ros group compared with the HFD group ( $\mathrm{p}<0.05$; Fig. 8$)$.

\section{Discussion}

HCC typically has a poor prognosis, and the majority of patients are diagnosed with progressive liver cancer with a 5-year survival rate of approximately $2 \%$. Therefore, the 

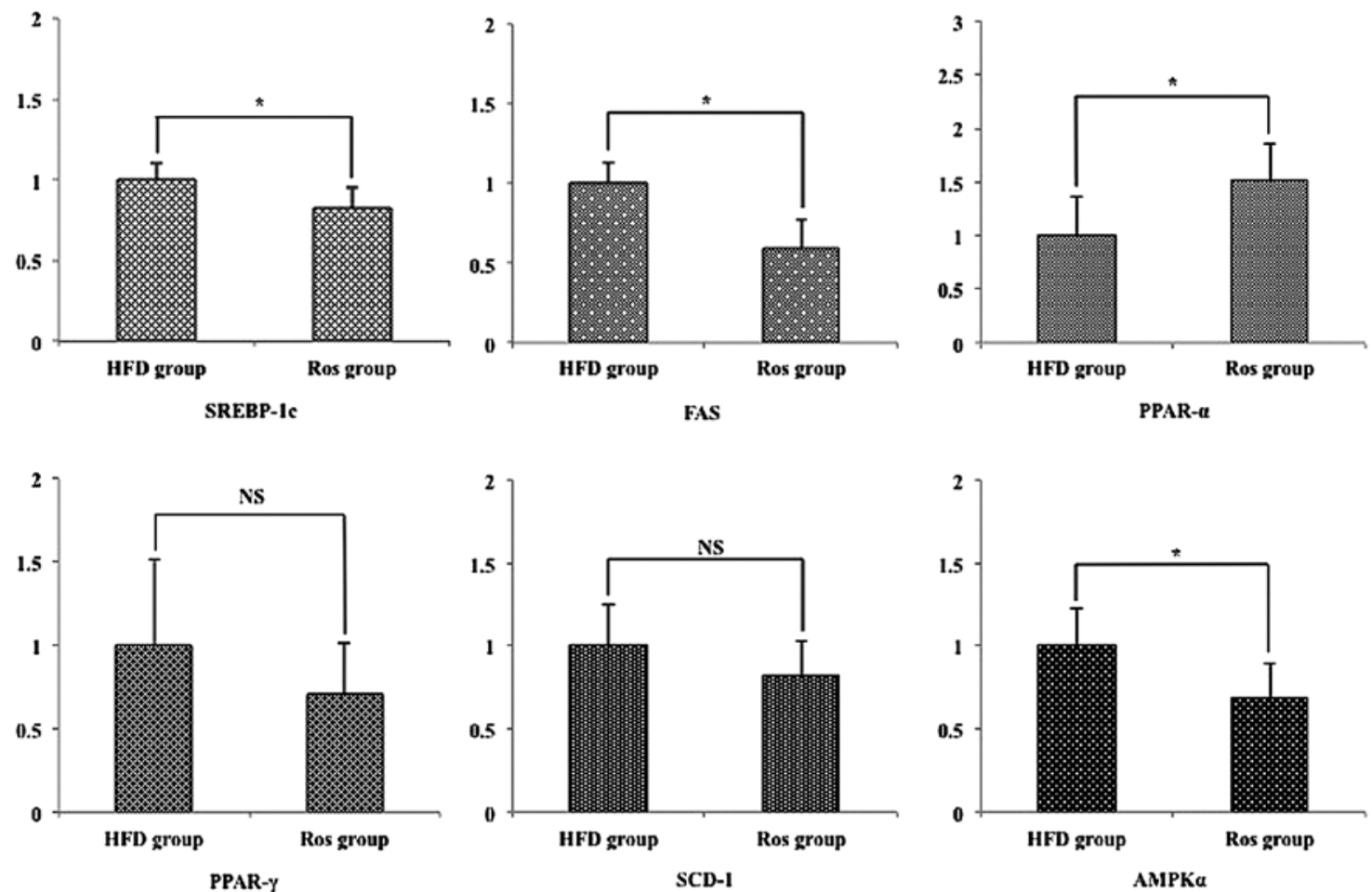

Figure 5. Relative hepatic sterol regulatory element binding protein-1c (SREBP-1c), fatty acid synthase (FAS), peroxisome proliferator-activated receptors (PPAR)- $\alpha$, PPAR- $\gamma$, stearoyl-CoA desaturase-1 (SCD-1) and AMP-activated protein kinase (AMPK) $\alpha$ levels in the mice in the high-fat diet (HFD) group and in the mice in the rosuvastatin (Ros; fed the HFD supplemented with $0.00125 \%$ Ros) group were measured by RT-qPCR. The levels of the lipogenic-related genes, SREBP-1c, FAS and AMPK $\alpha$ were significantly decreased in the rosuvastatin (Ros) group compared with the HFD group ("p $<0.05$ ). In addition, the levels of PPAR- $\alpha$ were significantly increased in the Ros group compared with the HFD group ( $\mathrm{p}<0.05)$. However, there was no significant difference in the levels of PPAR- $\gamma$ between the 2 groups (NS, not significant).
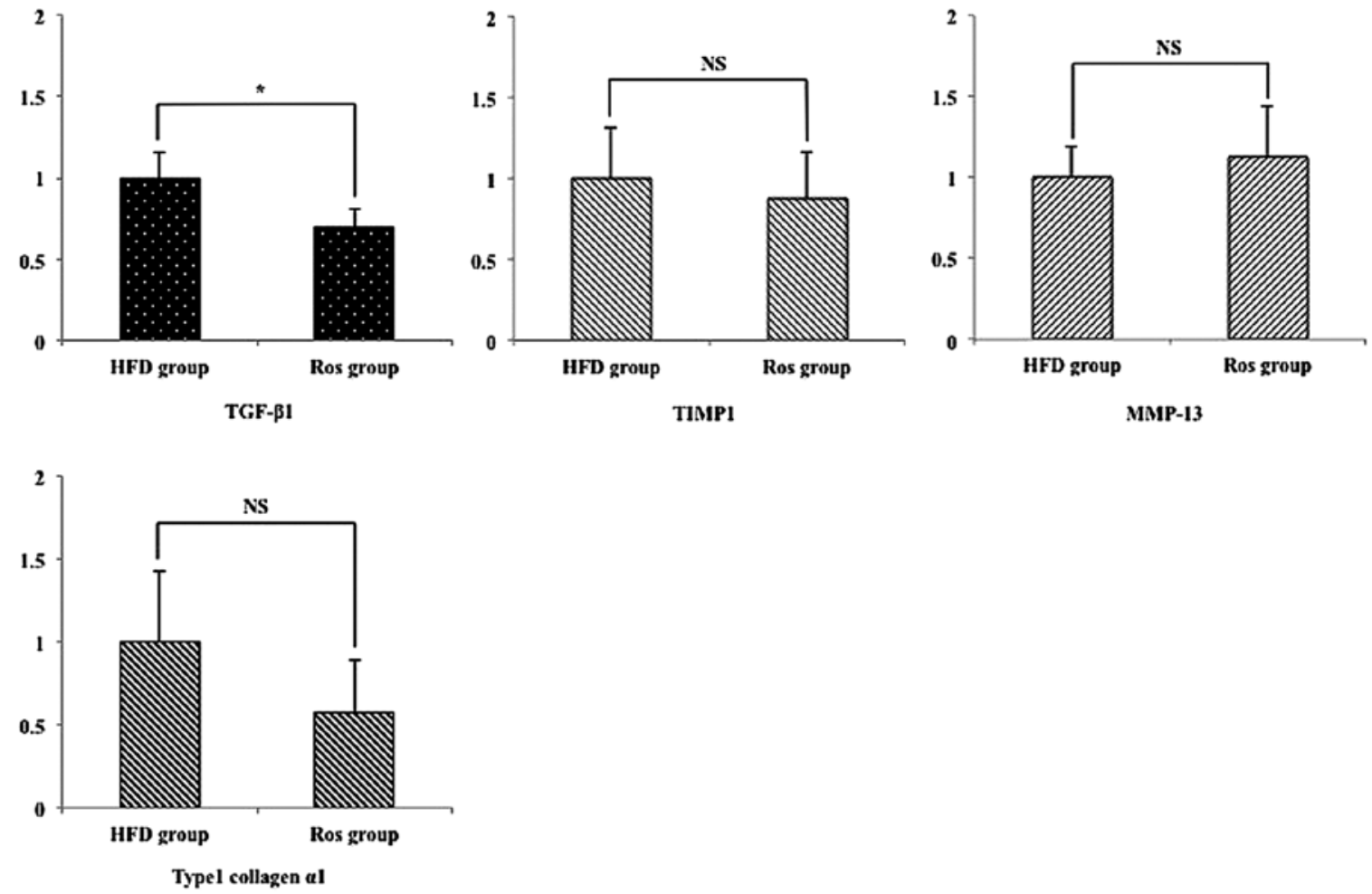

Figure 6. Relative hepatic transforming growth factor (TGF)- $\beta 1$, tissue inhibitors of matrix metalloproteinases (TIMP)-1, matrix metalloproteinases (MMP)-13 and type 1 collagen $\alpha 1$ levels in the mice in the high-fat diet (HFD) group and in the mice in the rosuvastatin (Ros; fed the HFD supplemented with $0.00125 \%$ Ros) group were measured by RT-qPCR. The levels of the profibrogenic gene, TGF- $\beta 1$, were significantly decreased in the Ros group ("p $<0.05)$. However, there was no significant difference in the expression levels of TIMP1, MMP-13 and type 1 collagen $\alpha 1$ between the 2 groups (NS, not significant). 

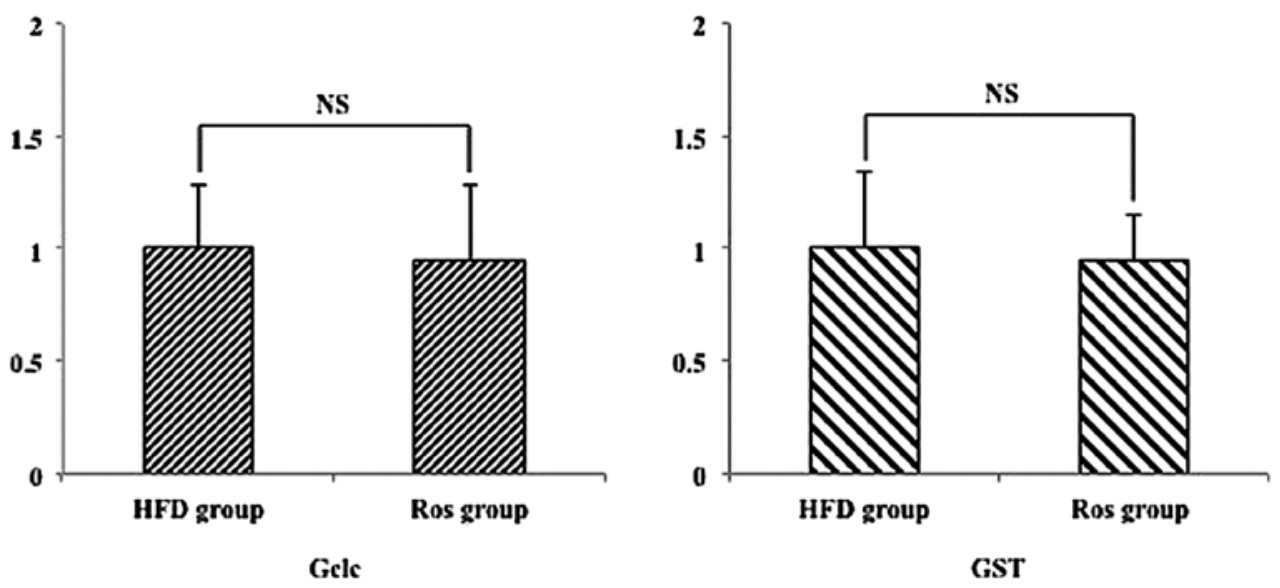

Figure 7. Relative hepatic glutamate-cysteine ligase, catalytic subunit (Gclc), and glutathione S-transferase (GST) levels in the mice in the high-fat diet (HFD) group and in the mice in the rosuvastatin (Ros; fed the HFD supplemented with $0.00125 \%$ Ros) group were measured by RT-qPCR. There was no significant difference in the expression levels of Gclc and GST between the 2 groups (NS, not significant).
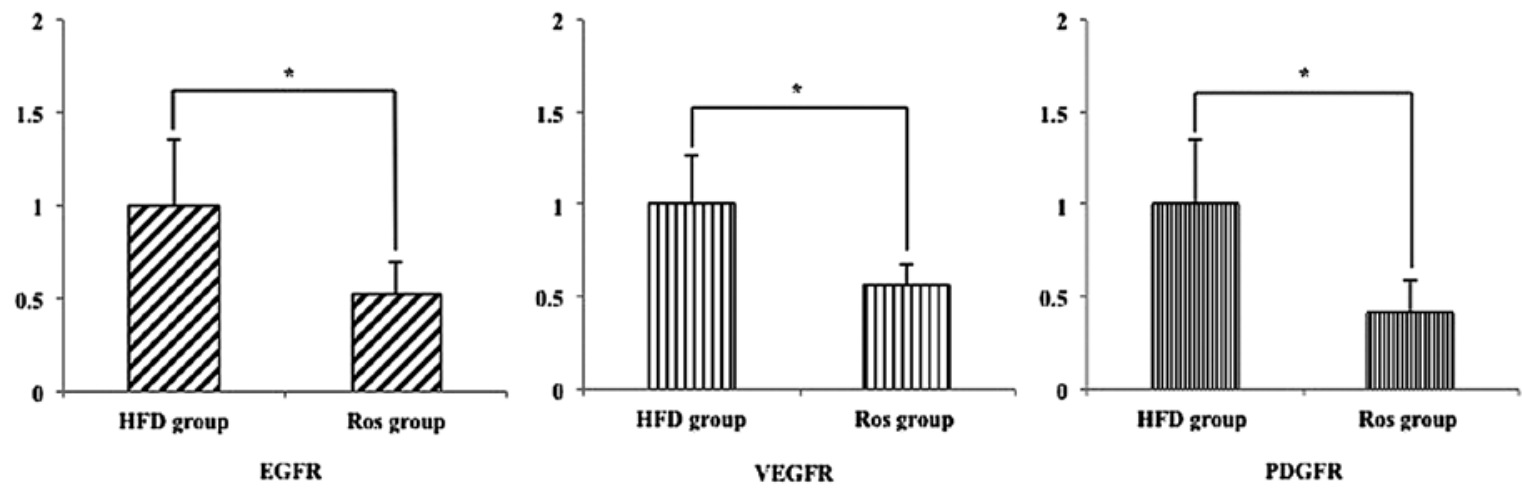

Figure 8. Relative hepatic epidermal growth factor (EGF), vascular endothelial growth factor (VEGF) and platelet-derived growth factors (PDGF) levels in the mice in the high-fat diet (HFD) group and in the mice in the rosuvastatin (Ros; fed the HFD supplemented with 0.00125\% Ros) group were measured by RT-qPCR. The levels of the hepatocellular carcinoma (HCC)-related genes, epidermal growth factor receptor (EGFR), vascular endothelial growth factor receptor (VEGFR) and platelet-derived growth factor receptor (PDGFR) were significantly decreased in the Ros group compared with the HFD group ("p $<0.05$ ),

prevention of HCC is important for reducing its mortality and morbidity. It is considered necessary to identify patients at risk of the occurrence of HCC and to develop a safe chemopreventive agent. Hyperlipidemia is one of the risk factors responsible for the occurrence of NASH, which ultimately causes HCC. An increase in the extent of hyperlipidemia translates to an increase in the risk of the occurrence of HCC. Ros is a hypolipidemic agent. In addition to its anti-hyperlipidemic effect, it has been shown to exert anti-inflammatory and anti-arteriosclerotic effectsin cardiovascular diseases both in vivo and in vitro (4-6). Although Ros is commonly used in hyperlipidemic patients, no study has been reported to date on the effect of the drug on the occurrence of $\mathrm{HCC}$ in the presence of an underlying NASH, at least to the best of our knowledge. The present study showed the potential protective effect of Ros against HCC or tumor formation. Our data also showed that this protective effect is not only partially mediated by its anti-inflammatory effect and the downregulation of genes involved in new lipogenesis, but may also be mediated by the downregulation of vascular proliferative factors.

It has beent demonstrated that Ros exerted anti-inflammatory effects not only in cardiovascular diseases, but also on factors involved in the occurrence and progression of NASH (4-6). These findings are consistent with the biochemistry measurements of AST and ALT in our study. In fibrogenesis, however, Ros exerted its effect on TGF- $\beta 1$, but not on stellate cell-derived factors.

Our results also suggested that Ros exerted its effect on the expression of lipid-related genes. Biochemical analyses revealed that the serum and liver TG levels decreased in the Ros group. To elucidate the mechanism involved, we measured the hepatic expression of some lipogenic and lipid oxidation-related genes in both groups. The results of RT-qPCR assay confirmed that the administration of Ros significantly affected the expression of a number of lipid-related genes. In particular, SREBP-1c (23), a key transcription activator in hepatic lipid synthesis, was significantly decreased in the Ros group, compared with the HFD group. The multi-subunit enzyme, AMPK, is recognized as a major regulator in the hepatic lipid synthesis pathway and fatty acid oxidation $(24,25)$. We found that the expression of the AMPK $\alpha 1$ subunit was higher in the Ros group than in the HFD group. The molecule PPAR- $\alpha$ is a member of the PPAR subfamily of nuclear receptors that transcriptionally promotes peroxisomal, microsomal and mitochondrial oxidation (17). 
PPAR- $\alpha$ expression in the liver was significantly higher in the Ros group than in the HFD group. By contrast, no significant difference was observed between the two groups as regards the expression of PPAR- $\gamma$ (18), another member of the PPAR subfamily, which transcriptionally initiates adipocyte differentiation.

It has previously been shown that the administration of eicosapentaenoic acid (EPA), a hypolipidemic agent, to Pten knockout mice induces the expression of antioxidant genes and inhibits the formation of reactive oxygen species. Thus, we considered whether Ros exerts an antioxidant effect such as EPA. In our study, no significant expression of antioxidant genes, such as Gclc and GST, was induced in the livers of mice treated with Ros (20). These findings suggest that Ros has no antioxidant effect on STAM mouse livers.

Importantly, Ros suppressed the occurrence of hepatic adenoma and HCC in the STAM mice used in this study. While this effect has been reported for EPA (26), it has not even investigated for Ros. EPA has been reported to have an antitumor effect originating from cell death induction and cell growth inhibition. In addition to focusing our attention to the already demonstrated effect of Ros on cardiovascular diseases, we investigated the expression of vascular proliferative factors, based on the fact that sorafenib, the only oral treatment for progressive HCC, has a suppressive effect on tumor vessel proliferation. Our results indicated that the expression of vascular proliferative factors, including EGF, VEGF and PDGF, was inhibited by almost 50\% in the Ros group, compared with the HFD group. This finding suggests that the hypolipidemic agent, Ros, may have an antitumor effect, in addition to its antihyperlipidemic effect, against HCC associated with NASH.

Ros is a drug used in the treatment of many hyperlipidemic patients. In the present study, we demonstrated that the administration of Ros is a potential treatment for NASH and its final stage, HCC. We suggest that the effects of Ros be evaluated in future prospective and retrospective randomized placebocontrolled clinical studies.

\section{Acknowledgements}

The authors would like to thank Yukio Nakahira and Eiko Koubayashi, at the Osaka Medical College, for providing them with technical support.

\section{References}

1. El-Serag HB and Rudolph KL: Hepatocellular carcinoma: Epidemiology and molecularcarcinogenesis. Gastroenterology 132: 2557-2576, 2007.

2. Parkin DM, Bray F, Ferlay J and Pisani P: Global cancer statistics, 2002. CA Cancer J Clin 55: 74-108, 2005.

3. Ludwig J, Viggiano TR, McGill DB and Oh BJ: Nonalcoholic steatohepatitis: Mayo Clinic experiences with a hitherto unnamed disease. Mayo Clin Proc 55: 434-438, 1980.

4. Aviram M, Rosenblat M, Bisgaier CL and Newton RS: Atrovastatin and gemfibrozil metabolities, but not the parent drugs are potent antioxidants against lipoprotein oxidation. Antherosclerosis 138: 272-280, 1998.

5. Ridker PM, Cannon CP, Morrow D, Rifai N, Rose LM, McCabe $\mathrm{CH}$, Pfeffer MA and Braunwald E; Pravastatin or Atorvastatin Evaluation and Infection Therapy-Thrombolysis in Myocardial Infarction 22 (PROVE IT-TIMI 22) Investigators: C-reactive protein levels and outcomes after statin therapy. N Engl J Med 352: 20-28, 2005.
6. Laufs U, La Fata V, Plutzky J and Liao JK: Upregulation of endothelial nitric oxide synthase by HMG CoA reductase inhibitors. Circulation 97: 1129-1135, 1998.

7. Nezasa K, Higaki K, Matsumura T, Inazawa K, Hasegawa $H$, Nakano $M$ and Koike M: Liver-specific distribution of rosuvastatin in rats: Comparison with pravastatin and simvastatin. Drug Metab Dispos 30: 1158-1163, 2002.

8. Ikeda Y, Young LH and Lefer AM: Rosuvastatin, a new HMG-CoA reductase inhibitor, protects ischemic reperfused myocardium in normocholesterolemic rats. J Cardiovasc Pharmacol 41: 649-656, 2003.

9. Bulhak A, Sjoquist PO and Pernow J: Rosuvastatin protects the myocardium against ischaemia-reperfusion injury via inhibition of GGPP synthesis. Cardiovasc J S Afr 15: S11, 2004.

10. Weinberg EO, Scherrer-Crosbie M, Picard MH, Nasseri BA, MacGillivray C, Gannon J, Lian Q, Bloch KD and Lee RT: Rosuvastatin reduces experimental left ventricular infarct size after ischemia-reperfusion injury but not total coronary occlusion. Am J Physiol Heart Circ Physiol 288: H1802-H1809, 2005.

11. Bulhak AA, Gourine AV, Gonon AT, Sjöquist PO, Valen G and Pernow J: Oral pre-treatment with rosuvastatin protects porcine myocardium from ischaemia/reperfusion injury via a mechanism related to nitric oxide but not to serum cholesterol level. Acta Physiol Scand 183: 151-159, 2005.

12. Takakura K, Koido S, Fujii M, Hashiguchi T, Shibazaki Y, Yoneyama H, Katagi H, Kajihara M, Misawa T, Homma S, et al: Characterization of non-alcoholic steatohepatitis-derived hepatocellular carcinoma as a human stratification model in mice. Anticancer Res 34: 4849-4855, 2014.

13. Gao B: Innate immunity and steatohepatitis: A critical role of another toll (TLR-9). Gastroenterology 139: 27-30, 2010.

14. Horton JD, Shah NA, Warrington JA, Anderson NN, Park SW, Brown MS and Goldstein JL: Combined analysis of oligonucleotide microarray data from transgenic and knockout mice identifies direct SREBP target genes. Proc Natl Acad Sci USA 100: 12027-12032, 2003.

15. Foretz M, Ancellin N, Andreelli F, Saintillan Y, Grondin P, Kahn A, Thorens B, Vaulont S and Viollet B: Short-term overexpression of a constitutively active form of AMP-activated protein kinase in the liver leads to mild hypoglycemia and fatty liver. Diabetes 54: 1331-1339, 2005.

16. Cohen P and Friedman JM: Leptin and the control of metabolism: Role for stearoyl-CoA desaturase-1 (SCD-1). J Nutr 134: 2455S-2463S, 2004.

17. Yu S, Rao S and Reddy JK: Peroxisome proliferator-activated receptors, fatty acid oxidation, steatohepatitis and hepatocarcinogenesis. Curr Mol Med 3: 561-572, 2003.

18. Tontonoz P, Hu E, Graves RA, Budavari AI and Spiegelman BM: mPPAR gamma 2: Tissue-specific regulator of an adipocyte enhancer. Genes Dev 8: 1224-1234, 1994.

19. Friedman SL: The cellular basis of hepatic fibrosis. Mechanism and treatment strategies. N Engl J Med 328: 1828-1835, 1993.

20. Gorrini C, Harris IS and Mak TW: Modulation of oxidative stress as an anticancer strategy. Nat Rev Drug Discov 12: 931-947, 2013.

21. Chaparro M, González Moreno L, Trapero-Marugán M, Medina J and Moreno-Otero R: Review article: Pharmacological therapy for hepatocellular carcinoma with sorafenib and other oral agents. Aliment Pharmacol Ther 28: 1269-1277, 2008.

22. Siegelin MD, Raskett CM, Gilbert CA, Ross AH and Altieri DC: Sorafenib exerts anti-glioma activity in vitro and in vivo. Neurosci Lett 478: 165-170, 2010.

23. Tontonoz P, Kim JB, Graves RA and Spiegelman BM: ADD1: A novel helix-loop-helix transcription factor associated with adipocyte determination and differentiation. Mol Cell Biol 13: 4753-4759, 1993

24. Hardie DG and Carling D: The AMP-activated protein kinase-fuel gauge of the mammalian cell? Eur J Biochem 246: 259-273, 1997.

25. Winder WW and Hardie DG: AMP-activated protein kinase, a metabolic master switch: Possible roles in type 2 diabetes. Am J Physiol 277: E1-E10, 1999.

26. Ishii H, Horie Y, Ohshima S, Anezaki Y, Kinoshita N, Dohmen T, Kataoka E, Sato W, Goto T, Sasaki J, et al: Eicosapentaenoic acid ameliorates steatohepatitis and hepatocellular carcinoma in hepatocyte-specific Pten-deficient mice. J Hepatol 50: 562-571, 2009. 